University of Nebraska - Lincoln

DigitalCommons@University of Nebraska - Lincoln

Robert Katz Publications

Research Papers in Physics and Astronomy

June 1968

\title{
Width of Heavy-Ion Tracks in Emulsion
}

E. J. Kobetich

University of Nebraska-Lincoln

Robert Katz

University of Nebraska-Lincoln, rkatz2@unl.edu

Follow this and additional works at: https://digitalcommons.unl.edu/physicskatz

Part of the Physics Commons

Kobetich, E. J. and Katz, Robert, "Width of Heavy-Ion Tracks in Emulsion" (1968). Robert Katz Publications. 8.

https://digitalcommons.unl.edu/physicskatz/8

This Article is brought to you for free and open access by the Research Papers in Physics and Astronomy at DigitalCommons@University of Nebraska - Lincoln. It has been accepted for inclusion in Robert Katz Publications by an authorized administrator of DigitalCommons@University of Nebraska - Lincoln. 


\title{
Width of Heavy-Ion Tracks in Emulsion*
}

\author{
E. J. Kobetich AND Robert Katz \\ Behlen Laboratory of Physics, University of Nebraska, Lincoln, Nebraska
}

(Received 18 December 1967)

\begin{abstract}
Profiles of the solid core of long, flat, ending tracks of heavy primary cosmic rays in Ilford G-5 emulsion have been measured by manually tracing their enlarged $(3500 \times)$ photographic image. These measurements agree well in the ending $3000 \mu$ of residual range $(\beta<0.3)$ with a reformulated theory of track width based on computation of the spatial distribution of ionization energy. The measured core width in G-5 emulsion is the sum of the sensitized cylinder diameter, at which $6000 \mathrm{erg} / \mathrm{cm}^{3}$ of ionization energy is deposited by $\delta$ rays, and the diameter of a developed grain.
\end{abstract}

\section{INTRODUCTION}

$\mathbf{I}^{\mathrm{N}}$ $\mathrm{N}$ 1965, Katz and Butts ${ }^{1}$ showed that the distribution of ionization energy, about the path of an energetic ion, provided a suitable basis for analyzing the core width of its track in electron sensitive emulsion. In the intervening time, a number of the components of the calculation have been improved substantially. The dissipation of the energy of normally incident electrons in plane films has been reformulated empirically ${ }^{2}$ so as to eliminate the original assumptions of a power-law range-energy relation, and of straight-line electron paths. The $\delta$-ray distribution formula has been amended to include atomic binding effects. Similar progress has been made in track measurement through use of photomicrographic techniques. The result has been a considerable improvement in the agreement between theory and experiment, to the point that the theory describes measured tracks well, to a residual range of about $3000 \mu(\beta<0.3)$. The principal conclusion of the earlier work, that the track core is defined by the cylinder diameter at which the deposited ionization energy

\footnotetext{
* Supported by the U.S. Atomic Energy Commission and the National Science Foundation.

1 R. Katz and J. J. Butts, Phys. Rev. 137, B198 (1965).

2 E. J. Kobetich and R. Katz, third preceding paper, Phys. Rev. 170, 391 (1968).
}

is $6000 \mathrm{erg} / \mathrm{cm}^{3}$, in Ilford G-5 emulsion, remains unchanged. The theory approximates the emulsion as a homogeneous medium, treats the photographic grain as a point particle, and employs the concept of an energy-deposition threshold for the sensitization of a photographic grain. The validity of these approximations improves as the ratio of the diameter of the sensitized cylinder to the grain diameter increases, so that the theory provides a basis for the identification of ending tracks of very heavy primary cosmic rays in G-5 emulsion.

\section{TRACK MEASUREMENT AND CALIBRATION}

Two stacks of Ilford G-5 emulsion flown at balloon altitudes from Ft. Churchill at different times, and processed in different laboratories to approximately the same grain diameter $(0.7 \mu)$ were scanned for long, flat, wide, ending tracks with a Bausch and Lomb StereoZoom microscope at $40 \times$. Track segments $50 \mu$ long were photographed, under photometric control, onto 100-ft rolls of 70-mm Kodak LS Pan film with a Leitz microscope equipped with a xenon lamp and a $100 \times$ Leitz Plano objective. Film rolls were processed and enlarged commercially onto 8-in.-wide rolls of Kodak Velox Premier paper, under photometric control, to a resultant enlargement of $3500 \times$. 


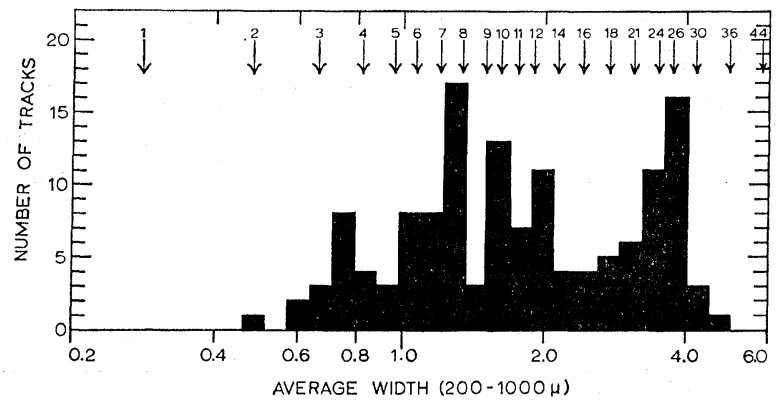

FIG. 1. Distribution of mean normalized track width in the residual range $200-1000 \mu$ found in two stacks of G-5 emulsion flown from Ft. Churchill and selectively scanned for long, flat, heavy tracks. The relative abundance of tracks at a mean width of $3.8 \mu$ identifies these tracks as due to iron nuclei.

To find the average track width, the photographic print was traced manually, with pen and ink, around the track core, ignoring clumps of silver grains not attached to the track. One grain of each isolated $\delta$ ray protruding from the track core was considered part of the track. Because of roughness of the track boundary, the width was determined by measuring the areas of specified segments with a planimeter. The total time required for a segment measurement was less than $5 \mathrm{~min}$, comparable to the time for an equivalent photometric measurement. Segment lengths for reporting the track width at specified ranges were so chosen that the average width did not change appreciably in the measured interval. In the ending $100 \mu$ the segments were $10 \mu$ long. Between $100 \mu$ and $1000 \mu$ the segments were $50 \mu$ long. At greater ranges, the segments were $100 \mu$ long.

Variations arising from changes in the contrast of the printing paper, from multiple printing from the same negative, and minor variations in focus when the tracks were rephotographed, yielded minor differences in track width which could not be distinguished from the variation in area measurement from observer to observer. Track photographs obviously out of focus were consistently traced as narrower than sharply focused photographs of the same track segment.

Range measurements were made with a dial micrometer attached to the microscope stage. Tracks were aligned parallel to the translational axis of the stage with a magnetically geared rotating superstage. ${ }^{3}$

Different observers read the location of the track edge somewhat differently. To accommodate for these differences in judgement, and to determine the mean diameter of a developed grain, the ending $30 \mu$ of each track was used to compensate each observer's measurement of the remainder of the track. The mean width in the $0-30-\mu$ interval was subtracted from the mean width in all other segments to produce a "normalized track

${ }^{3}$ R. Katz, Rev. Sci. Instr. 37, 1601 (1966). width." When traced by $2-4$ observers, prints from a single negative were consistent to $5 \%$ in mean normalized track width. Since the track width is nearly proportional to $Z$, this implies a measurement uncertainty of $5 \%$ in $Z$ by track-width methods.

In the ending $30 \mu$, the track width is very weakly dependent on the atomic number of the ion responsible for the track formation. In the region of the broad maximum of the track width, the width is most sensitive to $Z$. This region of the track is rough in outline because a relatively small number of $\delta$ rays make up the track edge. To compensate for statistical fluctuation in the location of the boundary of the track core, track segment areas were accumulated in the interval 200 to $1000 \mu$ to yield a mean track width in this interval for ultimate $Z$ identification, by comparison with theory.

The distribution in mean track widths in the interval 200-1000 $\mu$, of the tracks selected for this investigation, is shown in Fig. 1. The abundant group at 3.8- $\mu$ mean width was identified as iron, from cosmic abundances. The $Z$ assignments shown by arrows at the top of the figure arise from the track-width theory. The distribution of Fig. 1 provides the only available calibration point for the theory.

Comparison of the widths of cosmic-ray tracks with those produced by known ions in G-5 emulsion at the Berkeley HILAC yielded inconsistent and inconclusive results. Machine-accelerated ions at $10 \mathrm{MeV}$ /amu have a range less than $150 \mu$ and, when suitably oriented for photography, lie in the top $15 \mu$ of emulsion, while the cosmic-ray tracks used here were selected so as to lie near the middle of a $400-\mu$ or a $600-\mu$ pellicle. Similar discrepancies in photometrically measured track width have been observed by Hall and Zaffarano ${ }^{4}$ in the outer $15 \mu$ of emulsion.

Other difficulties attend the calibration problem. Inconsistencies in $\delta$-ray counting in ending tracks have led to the rejection of this technique as a basis for

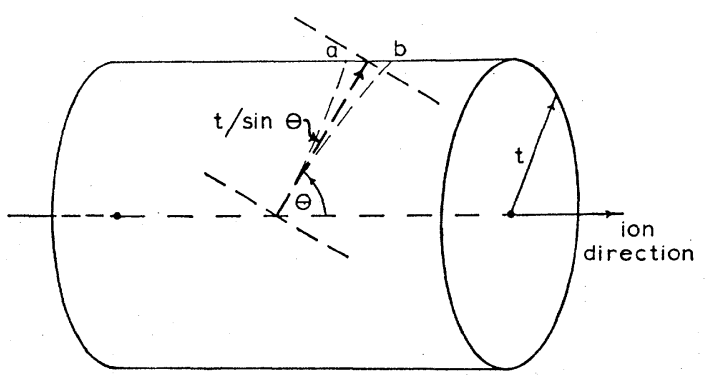

FIG. 2. The energy transmission of electrons, ejected at an angle $\theta$ to the ion's path, through a cylinder of radius $t$, is assumed to be equal to the transmission of these electrons through a slab of thickness $t / \sin \theta$. Differences between the cylindrical and slab geometry encountered by electrons scattered into path a are compensated in part by electrons scattered into path $\mathrm{b}$.

${ }^{4}$ J. E. Hall and D. J. Zaffarano, Nucl. Instr. Methods 48, 141 (1967) 

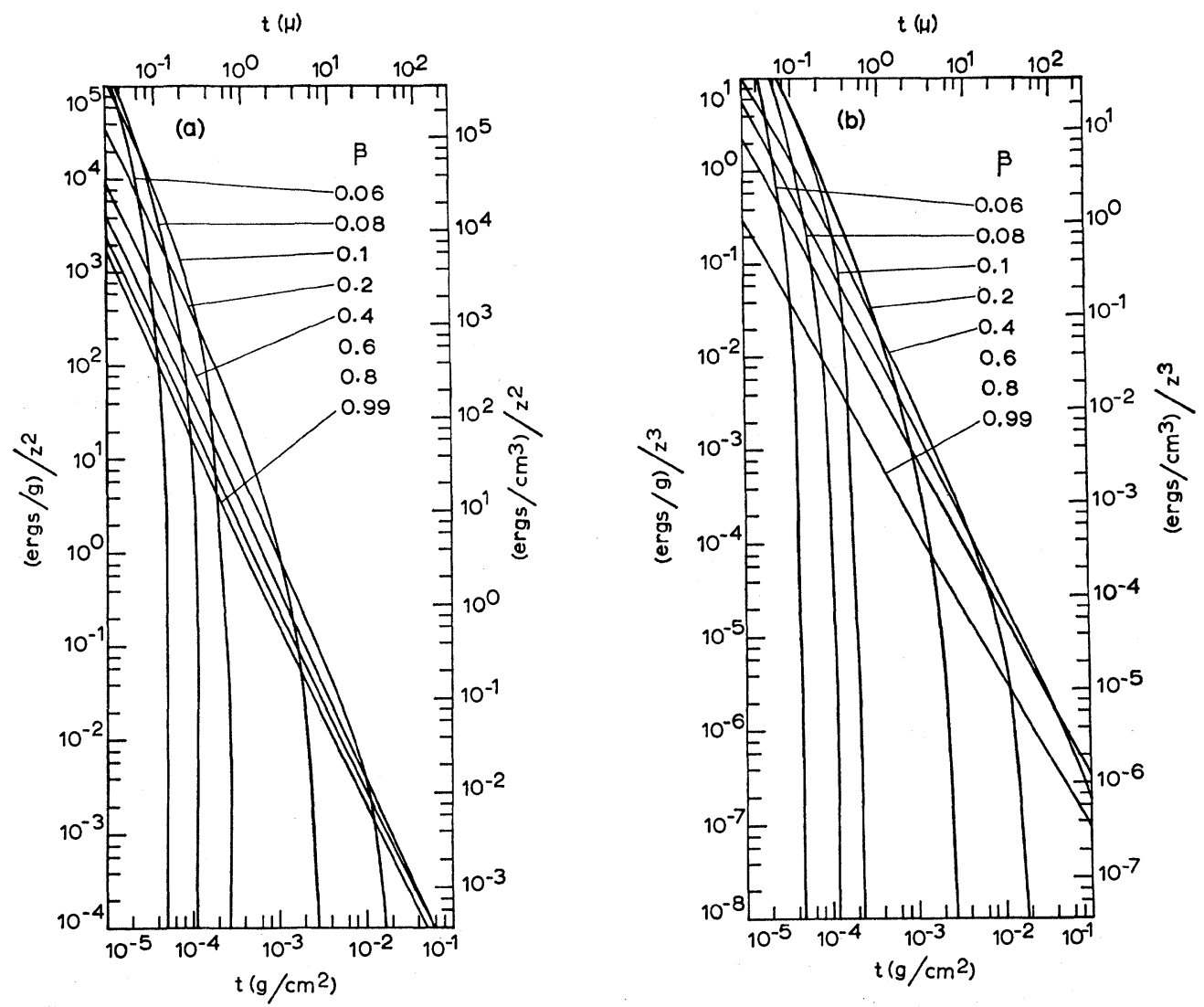

FIG. 3. Spatial distribution of ionization energy in emulsion. To find the energy dosage deposited at radius $t$ by an ion of effective charge number $z$ moving at speed $\beta c$, the value given in curve (b) must be multiplied by $z$, added to the value obtained from (a), and the sum multiplied by $z^{2}$.

calibration. Uncertainties in the identification of the charges and energies of fragments have led to the rejection of heavy-ion breakups for this purpose. As in most other investigations of the heavy primary cosmic rays, the relative cosmic abundance of iron provides the needed calibration.

\section{THEORY OF TRACK WIDTH}

As in several associated problems, ${ }^{5-7}$ the effects produced by the tangle of $\delta$ rays in the track core may be approximated as due to random processes. The response of the medium may be approximated through the cumulative Poisson distribution.

In their computer simulation of a latent image model of the photographic medium, Hamilton and Bayer ${ }^{8}$ showed that some 30-100 events are required to produce a latent image in a photographic grain. Since the cumu-

5 J. J. Butts and R. Katz, Radiation Res. 30, 855 (1967).

6 R. Katz and E. J. Kobetich, first preceding paper, Phys. Rev. 170, 401 (1968).

7 R. Katz and E. J. Kobetich, second preceding paper, Phys. Rev. 170, 397 (1968).

${ }^{8}$ J. F. Hamilton and B. E. Bayer, J. Opt. Soc. Am. 55, 528 (1965). lative Poisson distribution approaches a step function as the number of hits increases, the required dosage for sensitization of the track core has been taken to have a threshold or step-function character for the present work. The validity of this approximation is abetted by the rapid drop in deposited energy with distance from the ion's path.

The track-width theory is based on the computation of the spatial distribution of ionization energy, from empirical relations describing the dissipation of the energy of normally incident beams of electrons onto films of material, and on the $\delta$-ray distribution formula, empirically modified to take electron binding into account. ${ }^{2}$ The emulsion is approximated as a grainless, homogeneous medium of appropriate composition.

Since track diameters are measured in microns, the $\delta$ rays making up the track core have energies measured in $\mathrm{keV}$, and cannot be approximated as arising from a grazing collision, except in the region of relativistic ion energies. As in the case of $\beta$ decay, the angular distribution arises from a 3-body interaction. The energy of the recoiling parent atom may be ignored, but not its momentum. In the absence of clear experimental or theoretical guidance, the angular distribution has been 


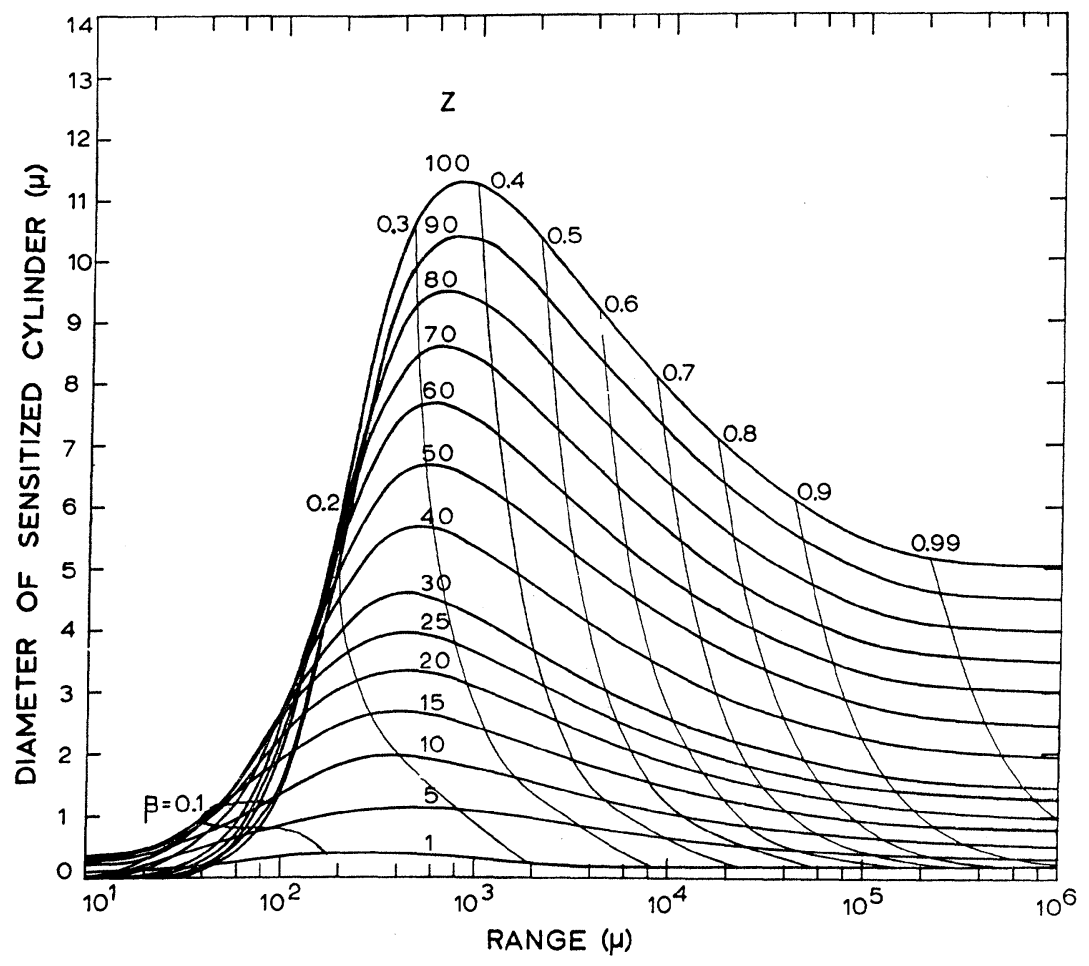

FIG. 4. Diameter of the sensitized cylinder in emulsion at which the energy dosage is $6000 \mathrm{erg} / \mathrm{cm}^{3}$. Energy deposition profiles are crossed by lines of constant $\beta$.

chosen to be the lowest normalized power of $\cos \theta$, forward, in the direction of the moving ion, which yields profiles in agreement with observation. The results of the present paper have been achieved by modifying the expression [Ref. 2, Eq. (5.6)] for the energy flux E through a cylinder of radius $t$ for normally ejected electrons to a form appropriate to the assumed angular distribution.

In order to use data obtained with slab geometry in the cylindrical geometry of the track, it is assumed that for all but the most heavily scattered electrons, it is as through the electrons are incident on a slab of thickness $t / \sin \theta$ (see Fig. 2), and that the effect of electrons which scatter so as to interact with too little material as compared to a slab, as in path a, which is compensated by those which encounter too much material, as in path $b$.

Using the notation of Kobetich and $\mathrm{Katz},{ }^{2}$ the energy flux $\mathbf{E}$, through a cylinder of radius $t$ may be found from the $\delta$-ray distribution formula which gives the number, $d n_{i} / d w$, of electrons per unit energy interval per unit path length ejected from atoms of type $i$ of the medium, the residual energy $W$ of an electron of initial kinetic energy $w$ after passing through a slab of thickness $t / \sin \theta$, the probability $\eta$ of such an electron being transmitted through the slab, and the angular distribution function $f(\theta)$. We write

$\mathbf{E}=\sum_{i=1}^{j} \int_{0}^{\pi / 2} \int_{w_{t}(\theta)}^{\omega_{m}-I_{i}} W(t, w, \theta) \eta(t, w, \theta) f(\theta)\left(d n_{i} / d w\right) d w$

$\times \sin \theta d \theta, \quad(1)$ where $I_{i}$ is the mean ionization potential of the $i$ th atom, $\omega_{m}$ is the maximum total energy which can be transferred in a collision with a free electron, and

$$
f(\theta)=5 \cos ^{4} \theta
$$

is the angular distribution empirically chosen to give best fit of theoretical and experimental track profiles. As before, ${ }^{2}$ the deposited energy density in a cylindrical shell is given by

$$
E=-(2 \pi t)^{-1} d \mathrm{E} / d t .
$$

Results of the numerical computation of Eq. (3) are shown in Fig. 3, in two graphs whose values must be combined to yield the deposited ionization energy, because of the form of the $\delta$-ray distribution formula. ${ }^{2}$ At ion speeds greater than $0.4 c$ the deposited ionization energy is insensitive to the assumed angular distribution. On the scale of the figure, the curves arising from an assumption of normal ejection at $\beta>0.4$ are indistinguishable from the plotted curves. Except where the plotted curves turn down, as demanded by the kinematic limit of $\delta$-ray energy, the density of deposited energy, $E$, varies directly with the square of the effective charge $z$ of the bombarding ion, and nearly inversely with the square of the distance $t$ from the ion's path. At constant $\beta$,

$$
E / z^{2} \sim 1 / t^{2} .
$$

The results given in Fig. 3 are suitable for comparison with track studies in emulsion, including photometric 
measurement of the density of the track profiles out to $200 \mu$ from the ion's path.

In order to produce theoretical track core profiles from Fig. 3, a threshold sensitivity for the emulsion must be assigned, $\beta$ must be translated into range, and the atomic number must be translated into effective charge.

The effective charge ze of an ion of atomic number $Z$, moving through matter at speed $\beta c$ has been given by Barkas $^{9}$ as

$$
z e=Z e\left[1-\exp \left(-125 \beta Z^{-2 / 3}\right)\right],
$$

while the range $R(\beta)$ of an ion of mass number $M$ and atomic number $Z$ is given in terms of the range $\lambda(\beta)$ of an ideal proton by Barkas and Berger ${ }^{10}$ as

where

$$
R(\beta)=M Z^{-2}\left[\lambda(\beta)+B_{Z}(\beta)\right],
$$

$$
\begin{aligned}
B_{Z}(\beta) & =1.525 \times 10^{-3} \beta Z^{5 / 3} & & \text { if } \beta \leqq 2 Z / 137 \\
& =2.233 \times 10^{-5} Z^{8 / 3} & & \text { if } \beta \geqq 2 Z / 137 .
\end{aligned}
$$

Best fit of theoretical and experimental track profiles results when the threshold energy for core formation is taken to be $6000 \mathrm{erg} / \mathrm{cm}^{3}$. The profiles of the diameter of the sensitized cylinder at $6000 \mathrm{erg} / \mathrm{cm}^{3}$ which result from the application of Eqs. (5) and (6) to Fig. 3 are shown in Fig. 4. The profiles are crossed by lines of constant $\beta$. The intersection of the profiles near $\beta=0.1$ arises from charge pickup.

To relate the computation from a hypothetical grainless emulsion to a real emulsion, we assume that a track edge will be formed by those grains of G-5 emulsion which receive an average does of $6000 \mathrm{erg} / \mathrm{cm}^{3}$. Ignoring the nonlinear variation in dosage across the grain volume, a grain whose center lies at the critical

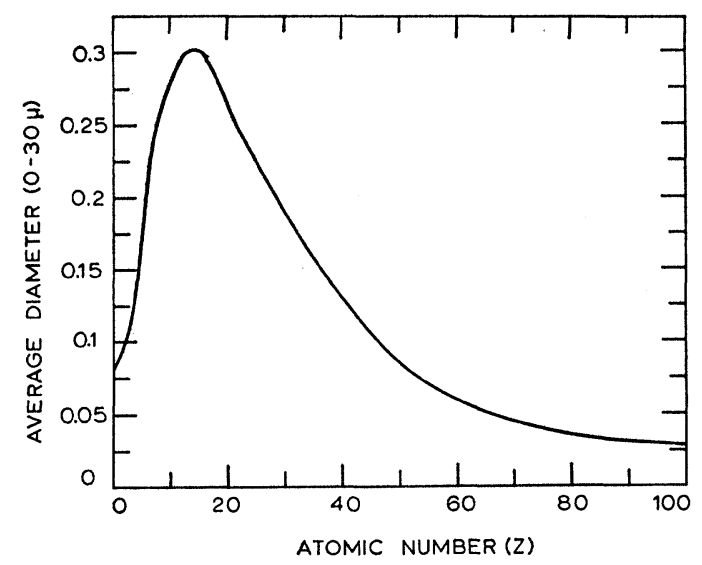

FIG. 5. Average diameter (in microns) of the $6000-\mathrm{erg} / \mathrm{cm}^{3}$ cylinder in the ending $30 \mu$.

\footnotetext{
${ }^{9}$ W. H. Barkas, Nuclear Research Emulsions (Academic Press Inc., New York, 1963), Vol. 1, p. 371.

${ }_{10}$ W. H. Barkas and M. J. Berger, Natl. Acad. Sci.-Natl. Res. Council, Publ. 1133, 103 (1964).
}

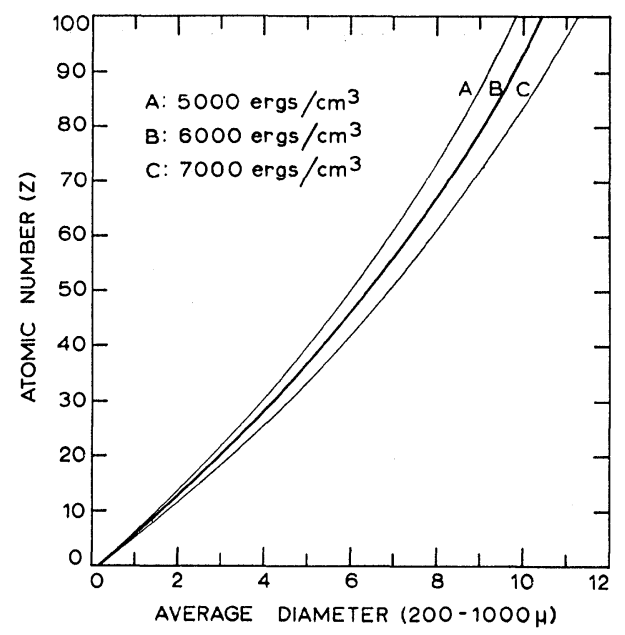

Fig. 6. Normalized average diameter $(200-1000 \mu)$ of the sensitized cylinder (in microns) as a function of $Z$ at (A) 5000, (B) 6000 , and (C) $7000 \mathrm{erg} / \mathrm{cm}^{3}$.

cylinder will be developed to form the track edge, thus adding the diameter of a developed grain to the diameter of the sensitized cylinder to make up the observed track width. In a similar computation of the cross sections for the interaction of heavy ions with enzymes and viruses, Butts and $\mathrm{Kat}^{5}$ have shown that molecularsize effects are small beyond about three molecular radii. Applying this result to the present work, it may be inferred that the assumption that the dose at the center of the undeveloped grain represents the mean dose over the grain volume is valid at distances greater than $\sim 1 \mu$ from the ion's path, and is therefore most suitable for describing the tracks of atoms whose atomic number is five or more.

In practice it is convenient to compare normalized experimental track width to normalized energy-deposition profiles, subtracting in both cases the mean width in the $0-30-\mu$ range interval. For experimental data, this normalization procedure subtracts away both the width of the sensitized core in this interval, and the grain diameter, while only the width of the sensitized core is subtracted from the theory. The calculated mean width of the sensitized core in the $0-30-\mu$ interval is shown in Fig. 5.

To identify the atomic number of a track producing ion, the normalized experimental mean track width in the $200-1000-\mu$ range interval is applied to Fig. 6, which gives the normalized average theoretical core diameter as a function of $Z$. In Fig. 6, curves arising from a critical-dosage assignment of (A) $5000 \mathrm{erg} / \mathrm{cm}^{3}$, (B) $6000 \mathrm{erg} / \mathrm{cm}^{3}$, and (C) $7000 \mathrm{erg} / \mathrm{cm}^{3}$ are shown, representing possible limits of error in assignment of the threshold sensitivity, arising from the uncertainty in calibration. The track profiles are not appreciably changed in form by these variations, though the atomic numbers to be associated with the profiles are altered as given in Fig. 6. The $Z$ identifications shown in Fig. 1, 


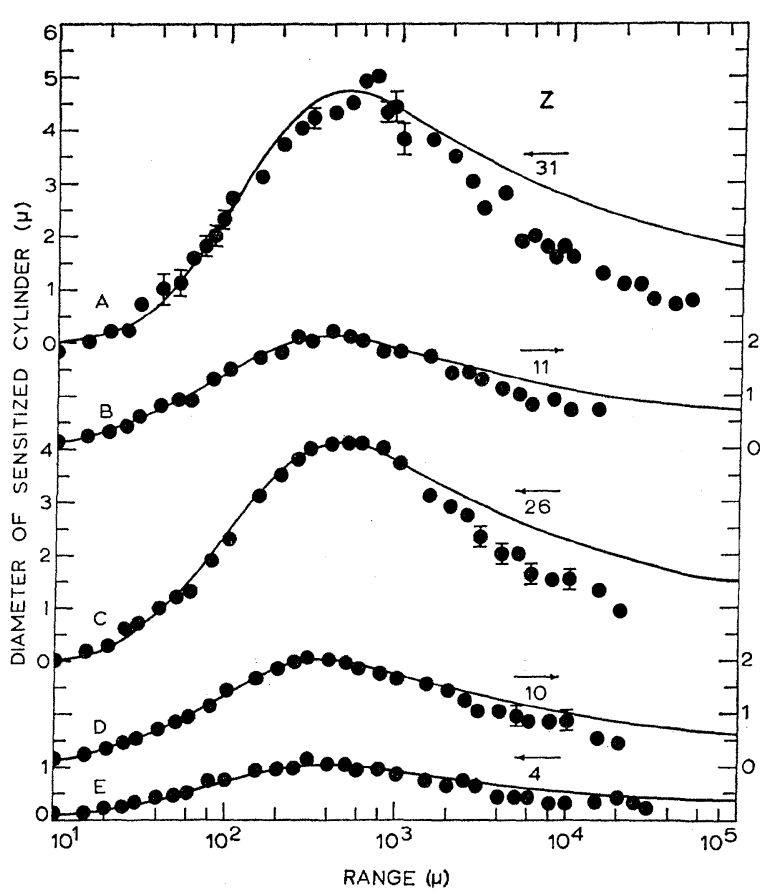

FIG. 7. Measured (and corrected) track widths compared to theoretical diameters of the sensitized cylinder. The lower three curves are average measurements from 10 tracks, while the upper two curves are each for a single track. Each track used lies in a single pellicle.

by arrows at the top of the figure, arise from curve $B$ in Fig. 6.

\section{EXPERIMENT VERSUS THEORY}

By restoring to the measured normalized track width the contribution of the sensitized core in the $0-30-\mu$ range interval, from Fig. 5, the results of track width measurement can be compared to the calculated energy deposition profiles, with typical results which are shown in Fig. 7. The points clustering about the profile $\mathrm{C}$ labeled $Z=26$ have been obtained by averaging the measurements of 10 tracks so identified. The same procedure was used for the profiles $\mathrm{D}$ and $\mathrm{E}$ labeled 10 and 4. Statistical variations in $\delta$-ray production are averaged out in this manner. The profile B labeled 11 has points plotted over it arising from nine measurements of a single track, four of which were on prints from one negative while five were on prints from a second negative. Points surrounding the theoretical $Z=31$ profile, $\mathrm{A}$, were obtained by a single observer who traced one print from each of two negatives. In all cases, error bars representing the mean deviation of the observations are shown, where these lie outside the plotted points. Each of the tracks used lies in a single pellicle.

Agreement between theory and experiment is excellent below a residual range of about $3000-4000 \mu$ $(\beta<0.3)$. At higher ion velocities the theory consistently overestimates the track width. Our attempts to resolve this discrepancy using a variety of reasonable assumptions combined with a point-particle theory have been unsuccessful. Nevertheless, some conclusions can be drawn about relativistic tracks, and the tracks of atoms of higher atomic number than have been measured here. The observed validity of the theory below $\beta=0.3$ implies that the measured width of the track of an ion of atomic number 100 is less than $12 \mu$ at its maximum width, and that the width of the track of such an ion at relativistic speeds is less than $6 \mu$, as implied by the energy deposition profiles, and may be as little as $3 \mu$, as implied by the discrepancy between theory and observation for the $Z=31$ track in Fig. 7, which is continuous for $5 \mathrm{~cm}$ in one pellicle and enters that pellicle at $\beta \simeq 0.83$.

There are independent experimental confirmations of the magnitude of the threshold dose.

The threshold dose for core formation of $6000 \mathrm{erg} / \mathrm{cm}^{3}$ corresponds to an averaged absorbed energy of $39 \mathrm{eV}$ in a volume of $0.275 \mu$, corresponding to the mean diameter of an undeveloped grain, as compared to the figure of $30 \mathrm{eV}$ for photon excitation given by Barkas. ${ }^{11}$

Integration of the $\beta=0.99$ curve of Fig. 3 over the volume of an undeveloped grain yields the energy delivered to a grain by a proton passing through its center as about $27 \mathrm{eV}$, roughly two standard deviations below the mean required energy for sensitization. This implies that the deposited energy/grain will exceed $39 \mathrm{eV}$ along the track of a relativistic proton over about $3 \%$ of the available grains. According to Shapiro ${ }^{12}$ a relativistic proton typically sensitizes about 20 grains $/ 100 \mu$, while the maximum grain density is 500 grains $/ 100 \mu .^{13}$

No information is available from Ilford regarding the response of its G-5 emulsion to $\mathrm{keV}$ electron bombardment. For a comparable material, Kodak NTB-2 emulsion, the manufacturer states ${ }^{14}$ that a normally developed emulsion achieves unit photographic blackness when bombarded with an average of 1 electron $/ \mu^{2}$ at $100 \mathrm{keV}$. This implies that the required dosage lies between 3500 and $6500 \mathrm{erg} / \mathrm{cm}^{3}$, depending on the fraction of electrons assumed to be back scattered from the glass support of the $25-\mu$ plates with which the data were obtained.

\section{TRACKS OF MAGNETIC MONOPOLES IN EMULSION}

The predicted diameter of the sensitized cylinder produced in emulsion by a magnetic monopole has been

${ }^{11} \mathrm{H}$. Barkas, Nuclear Research Emulsions (Academic Press Inc., New York, 1963), Vol. 1, p. 27.

12 M. M. Shapiro, in Encyclopedia of Physics, edited by S. Flügge (Springer-Verlag, Berlin, 1958), Vol. XLV, p. 356.

${ }_{13}$ H. Barkas, Nuclear Research Emulsions (Academic Press Inc., New York, 1963), Vol. 1, p. 397.

${ }^{14}$ Kodak Tech Bits, No. 2, Eastman Kodak Co., Rochester, N.Y. (unpublished). 
Fig. 8. Diameter of the sensitized cylinder at $6000 \mathrm{erg} / \mathrm{cm}^{3}$ produced by magnetic poles having pole strengths of 1,2 , and 4 Dirac monopoles $(g=137 e / 2)$, and masses of 3,6 , and $20 \mathrm{amu}$.

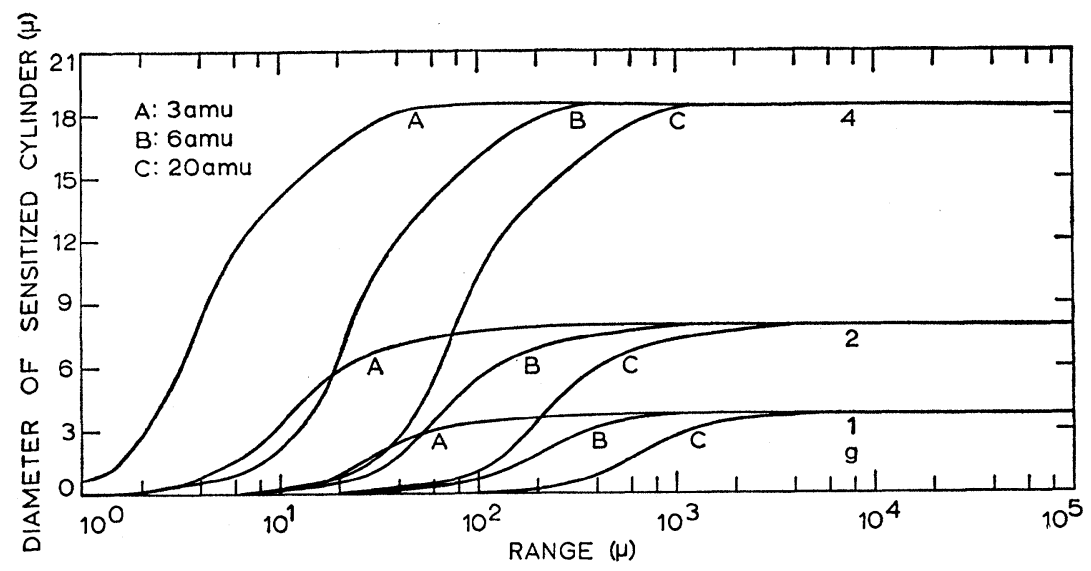

calculated from the assumption that the $\delta$ rays it produces are ejected normally, in the direction of its associated electric field. Results are shown in Fig. 8 for poles whose strength is 1,2 , and 4 times the unit Dirac monopole $(g=137 e / 2)$, and whose mass is 3,6 , and $20 \mathrm{amu}$. The track description agrees with earlier computations by Katz and Butts, ${ }^{1}$ and follows that computation in relating $\beta$ to range. The observation of long tracks of unvarying width, or of very wide track segments, implies the observation of a magnetic monopole.

\section{OBSERVATION OF EXTREMELY WIDE TRACKS IN EMULSION}

In a recent exposure of a stack of G-5 emulsion of large frontal area at balloon altitudes over Texas, where ions require speeds greater than $0.9 c$ to penetrate the magnetic cutoff, Fowler, Adams, Cowen, and Kidd ${ }^{15}$ have found some extremely wide tracks which they identified as due to ions having atomic numbers up to 90 by measurements of the photographic density at distances from 10 to $40 \mu$ from the ion's path.

Through the kindness of Professor Fowler, we have photographed and measured the two heavier tracks shown in Figs. 3 and 4 of Ref. 15, identified by Fowler et $a l . .^{15}$ as due to $Z=67$ and 90 ions. The tracks had dip

${ }^{15}$ P. H. Fowler, R. A. Adams, V. G. Cowen, and J. M. Kidd, Proc. Roy. Soc. (London) A301, 39 (1967). angles of $22.5^{\circ}$ and $40^{\circ}$, respectively, were in the second emulsion of the stack (separated from the outermost emulsion by $1 \mathrm{~mm}$ of $\mathrm{Pb}$ ), and may be assumed to be generated by ions moving at speeds somewhat under $0.9 c$. The mean diameters of the two tracks were 4.4 and $6 \mu$, respectively, consistent with the identifications of Fowler et al., and with our observations that measured sensitized cylinder diameters (width minus grain diameter, here taken to be $0.9 \mu$ ) of energetic ions lie somewhat below the calculated values, as shown in Fig. 7. Independent values of $Z$ cannot be assigned these relativistic ions from the present track-width theory.

\section{ACKNOWLEDGMENTS}

We thank M. W. Friedlander, (the late) D. E. Guss, and Harvey Goldberg for help in the preparation, flying, and processing emulsion stacks; H. H. Heckman for assistance in exposing emulsion to the HILAC beams; C. R. Criss for processing and printing track negatives; George Hofer for his studies of the influence of photography on track-width measurements; Rose Ann Roth, Chris Sorensen, Bob Solick, Bob Holmes, and Arvin Hernes for their help in scanning and in track-width measurement. We are indebted to P. H. Fowler for the opportunity to photograph and measure very heavy tracks in his emulsions, and to him and J. M. Kidd for discussions of their work. 